\title{
Wnt Signaling Mutants Have Decreased Dentate Granule Cell Production and Radial Glial Scaffolding Abnormalities
}

\author{
Cheng-Ji Zhou, Chunjie Zhao, and Samuel J. Pleasure \\ Department of Neurology, Programs in Neuroscience and Developmental Biology, University of California, San Francisco, California 94143-0435
}

LRP6 mutant mice have generalized defects in the Wnt/ $\beta$-catenin signaling pathway because of the crucial function of LRP6 as a Wnt signaling co-receptor (Pinson et al., 2000). We examined the hippocampal phenotype of single LRP6 mutant mice as well as LRP6/Lef1 double mutant mice. LRP6 mutants had reduced production of dentate granule neurons and abnormalities of the radial glial scaffolding in the forming dentate gyrus. These defects were more severe with the addition of a single Lef1 null allele to an LRP6 null background. Pyramidal cell fields were unaffected in the LRP6, Lef1, or double mutants. The dentate defects were accompanied by decreased numbers of mitotic precursors in the migratory pathway to the dentate and in the displaced proliferative zone in the dentate itself. At earlier gestational ages, there was a reduction in the number of dentate granule cell progenitors in the dentate ventricular zone before the emigration of the earliest differentiated granule neurons and precursors to form the dentate anlage.

Key words: dentate; development; granule cell; proliferation; Wnt; LRP6

\section{Introduction}

Wnts are secreted glycoprotein signaling molecules that regulate many developmental events in vertebrates and invertebrates. In mammals, previous studies showed that Wnt signaling regulates the development of the dorsal neural tube (Lee and Jessell, 1999). Loss of Wnt1 and Wnt3A in double mutants leads to failed expansion of dorsal spinal precursors and subsequent loss of cell types derived from these precursors (Ikeya et al., 1997). Whether Wnt signaling also directly regulates neural cell fate in mammals is debated, although recent data indicates a cell fate role in addition to the proliferation function in the vertebrate neural crest (Hari et al., 2002).

The dorsal region of the mammalian telencephalon generates the neocortex and hippocampus. These structures develop adjacent to the cortical hem, an abundant source of Wnt ligands (Grove et al., 1998). Wnt3A null mice have no medial cortical wall and lack an organized hippocampal formation (Lee et al., 2000), and Lef1 mutants (a transcription factor in the Wnt pathway) have a defect limited to the dentate gyrus (Galceran et al., 2000).

To understand the function of the $\mathrm{Wnt} / \beta$-catenin pathway in hippocampal development, we analyzed mice with mutations in LRP6. LRP6 is a ubiquitously expressed, required co-receptor for signaling in the Wnt/ $\beta$-catenin pathway but is not involved in non-canonical pathways (Tamai et al., 2000). Mice with null al-

Received Sept. 3, 2003; revised 0ct. 13, 2003; accepted Nov. 6, 2003.

This work was supported by funding to S.J.P. from National Institutes of Health Grants NS002208 and MH66084, the Burroughs Wellcome Foundation, the John Merck Fund, the Whitehall Foundation, and the National Alliance for Autism Research. We acknowledge Bill Skarnes, Kathy Pinson, and Rudi Grosschedl for mutant mice, and Robert Hevner for Tbr1 antisera, and John Rubenstein for discussions.

Correspondence should be addressed to Dr. Samuel Pleasure, Department of Neurology, University of California, San Francisco, 513 Parnassus Avenue, Room S268, San Francisco, CA 94143-0435. E-mail: samuelp@itsa.ucsf.edu. DOI:10.1523/JNEUROSCI.4071-03.2004

Copyright $\odot 2004$ Society for Neuroscience $\quad$ 0270-6474/04/240121-06\$15.00/0 leles of LRP6 have hypomorphic phenotypes resembling those of multiple Wnt ligands and have decreased Wnt $/ \beta$-catenin signaling (Pinson et al., 2000; Maretto et al., 2003). Therefore, these mice allow analysis of the function of $\mathrm{Wnt} / \beta$-catenin signaling in the developing hippocampus.

We show that LRP6 mutants have a dentate phenotype closely resembling that of Lef1 null mutants and that LRP6 alleles and Lef1 alleles are additive in their effect on the dentate granule lineage. Furthermore, both Lef1 and LRP6 mutants have disorganization and diminishment of the dentate gyrus radial glial scaffolding and the granule cell defects in these mice are associated with failed expansion of a pool of defined dentate granule precursor cells in the dentate ventricular zone at early stages of development.

\section{Materials and Methods}

Mouse breeding and genotyping. LRP6 mutants were genotyped by X-gal staining intensity or by limb morphology (Pinson et al., 2000). Approximately $50 \%$ of mutants were exencephalic, and these embryos were excluded from analysis. Lef1 mutants were genotyped by PCR using primers described previously (Galceran et al., 2000). The LRP6-/-; Lef1 $+/$ - and Lef1-/-;LRP6+/- mutants were generated by matings either between double heterozygotes or double heterozygotes and single heterozygotes. No double homozygotes (LRP6-/-;Lef1-/-) were obtained at E10.5 (at least 20 matings were examined). All animals were treated according to protocols approved by University of California at San Francisco.

Brdu labeling and counting. Pregnant mice were injected with bromodeoxyuridine (BrdU) intraperitoneally at $100 \mu \mathrm{g} / \mathrm{gm}$ body weight, and the embryonic brains were removed $1 \mathrm{hr}$ later. Multiple sections from approximately the same level were counted from each animal to derive average values for each animal, and at least three animals from each genotype were used for comparisons. To count different anatomic compartments, standardized boxes were drawn on the images, and positive cells were counted within that compartment. Error bars indicate the SEM, and statistical significance was determined using the Student's $t$ 
test. Because our semiquantitative analysis did not take into account any alterations in dentate volume, its sensitivity for small differences was probably reduced, however, given the large magnitude of the alterations that we found, this approach seemed reliable for documenting the large differences seen.

Tissue staining. Fresh-frozen embryo brains were sectioned at $7 \mu \mathrm{m}$ and then fixed with $4 \%$ paraformaldehyde. Rabbit antibodies to Prox 1 (1:5000) (Bagri et al., 2002), GFAP (1:1000; Chemicon, Temecula, CA), and mouse antibodies to BrdU (1:1000; Roche Products, Hertforshire, UK) and neuron-specific $\beta$ tubulin (TuJ1; 1:1000; Covance, Denver, PA) were used. The single or double-immunofluorescence labeling was detected with secondary antibodies conjugated to Alexa fluorochromes (Molecular Probes, Eugene, OR), followed by nuclear counterstaining with Hoechst 33258 (Sigma, St. Louis, $\mathrm{MO})$. In situ hybridization was performed as described previously (Pleasure et al., 2000).

\section{Results}

\section{LRP6 mutant mice have decreased} production of dentate granule neurons

To determine if LRP6 mutant mice had hippocampal defects resembling those of Wnt3A or Lef1 mutants, we began by examining markers that label developing hippocampal neurons in late gestation [embryonic day 18.5 (E18.5)] embryos. The T-box transcription factor Tbr1 is expressed in all neurons originating from the cortical ventricular zone and allowed us to examine the overall structure and size of the hippocampal subfields. Tbr1 staining showed that the subiculum and CA pyramidal fields were intact in LRP6 mutants and showed that the dentate gyrus in LRP6 mutants was underdeveloped, consisting of far fewer cells (Fig. 1A, $A^{\prime}$ ). EphA4 is a tyrosine kinase receptor that labeled the CA2 and CA3 layers as well as the entire dentate gyrus, and in LRP6 mutants it was apparent that the pyramidal cell labeling was intact but that much of the dentate gyrus staining was missing (Fig. $1 B, B^{\prime}$ ).

The dentate gyrus at late gestation consists primarily of four cell types: Cajal-Retzius cells, hilar pyramidal neurons (CA4 and CA3c), recently born dentate granule neurons, and immature precursor cells in the proliferative tertiary matrix (Amaral, 1978; Altman and Bayer, 1990). Reelin labels Cajal-Retzius cells, and expression of Reelin in mutant mice was normal except for the overall somewhat smaller size of the dentate gyrus (Fig. $1 C, C^{\prime}$ ). Math2 is a basic helix-loop-helix (bHLH) transcription factor selectively expressed in pyramidal neurons, including CA1-4 layers (Pleasure et al., 2000), and Math2 expression was unaffected in mutant animals (Fig. $1 D, D^{\prime}$ ). It is important to note that at this age the CA4 neurons, which by later gestational ages are scattered throughout the hilus and are labeled by Math2 (Pleasure et al., 2000), form an extension of the hilar portion of the CA3 layer at this prenatal age.

To examine granule cell development we stained for Prox1, a homeobox protein expressed selectively in granule cells and found that LRP6 $-/-$ mutants had decreased numbers of Prox $1+$ cells in the dentate gyrus (Fig. $1 E, E^{\prime}$ ). Neurogenin2 is another bHLH transcription factor expressed in immature precursors in the dentate gyrus (Pleasure et al., 2000), and the num- ber of Neurogenin 2 expressing cells in the dentate was markedly diminished in mutant animals (Fig. $1 F, F^{\prime}$ ). Our conclusion is that LRP6 - / - mice have normal patterning of the hippocampal primordium and production of most cell types in the hippocampus but have a severe defect in the production of dentate granule neurons and an accompanying decrease in the number of immature dentate precursor cells.

\section{LRP6 and Lef1 alleles are additive in their effect on dentate development}

Based on our understanding of the canonical Wnt signaling pathway, Lef1 and LRP6 should be in the same signaling pathway, and compound mutants would be useful to better understand the in vivo function of Wnt signaling in dentate granule cell production. The previous analysis of Lef1 mutants showed a moderate decrease in the number of dividing precursors in the dentate gyrus but not in the migratory pathway (Galceran et al., 2000), so, in addition to the severity of the dentate granule cell production defect, we analyzed the distribution of acutely dividing precursor cells in LRP6, Lef1, and LRP6/Lef1 double mutants.

In wild-type mice, Prox1 labeled a well formed dentate granule cell layer at E18.5, and acute BrdU labeling showed a broad distribution of dividing precursor cells migrating to populate the dentate gyrus (Fig. $2 A, A^{\prime}, A^{\prime \prime}$ ). In addition, Proxl staining was apparent in the migratory stream to the dentate, and there were small numbers of Prox1/BrdU double-labeled cells (Fig. $\left.2 A, A^{\prime}, A^{\prime \prime}\right)$. As noted above, LRP6-/- mice had a dramatic reduction in the number of Prox $1+$ cells both in the dentate and in the migratory route to the dentate (Fig. $2 B, B^{\prime \prime}$ ). There was also a decrease in the number of BrdU-labeled (and BrdU/Prox1 

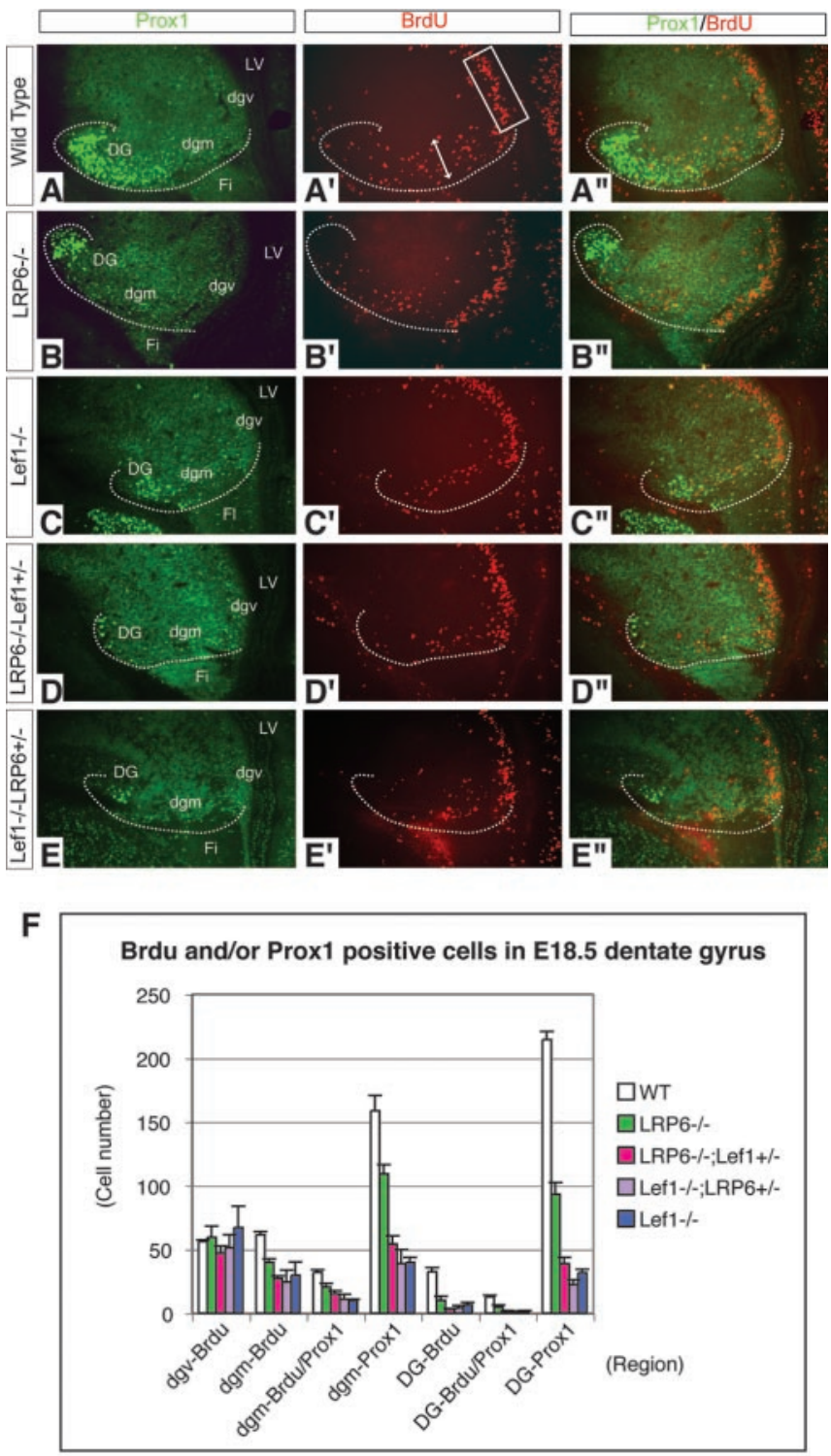

Figure 2. Loss of differentiated granule and precursor cells in E18.5 mutant mice. $A-E$ are stained with antibody to Prox1, $A^{\prime}-E^{\prime}$ are stained with antibody to $\mathrm{BrdU}$, and $A^{\prime \prime}-E^{\prime \prime}$ are the overlaid images. The outlines of the migratory route and dentate gyrus are indicated by the dotted line in each panel. In $A^{\prime}$, the arrow indicates the width of the migratory pathway. $F$ shows a graph of the counting data for cell types in each compartment. Error bars are SEM, and each data point is based on at least three animals of for each genotype. LV, Lateral ventricle; dgv, dentate gyrus ventricular zone; dgm, dentate gyrus migratory route; DG, dentate gyrus; Fi, fimbria.

double-labeled) cells in the migratory stream (Fig. $2 B^{\prime}, B^{\prime \prime}$ ). As described previously, Lef1-/- mutants had a similar, although more severe, loss of granule cells (Galceran et al., 2000), although our analysis did show that these mutants did have some Prox $1+$ cells in the dentate region, and in our hands there was also a dramatic loss of precursor cells in the dentate and migratory pathway (Fig. 2C, $C^{\prime}, C^{\prime \prime}$ ). LRP6-/-;Lef1+/- and Lef1-/-; LRP6+/ - mice showed even more severe loss of Prox1+ granule neurons and precursors (Fig. $\left.2 D, E, D^{\prime}, E^{\prime}, D^{\prime \prime}, E^{\prime \prime}\right)$. We never obtained LRP6-/-;Lef1 - / - embryos presumably because of early embryonic lethality. There did not appear to be any apparent decrease in the BrdU labeling in the ventricular zone at this same point.

We quantified these findings using sections from at least three animals of each genotype (Fig. 2F). LRP6-/- mutants had significant reductions in the number of precursors in the migratory path and in the dentate $(p<0.01)$. Furthermore, these mutants had approximately half the normal number of Prox $1+$ granule cells in the dentate and migratory pathway $(p<0.01)$. For the total number of Prox1+ cells in each compartment, Lef1-/mutants were statistically significantly more severely affected than LRP6-/ - mice $(p<0.01)$, whereas LRP6-/-;Lef1 +/mice were similar to Lef1-/- mice. The differences in BrdU+ precursor cells were of similar magnitude in all the genotypes when compared with wild-type, and there were not significant differences between the genotypes. Interestingly, we found no significant difference in the proliferation of precursor cells within the ventricular zone adjacent to the dentate gyrus at E17.5 in any genotype.

The lack of changes in proliferation in the ventricular zone adjacent to the forming dentate in late gestation implies that by this late stage of gestation cells in the ventricular zone are no longer producing granule cell neurons. In fact, previous studies of ours (using intraventricular retroviral injections) and others confirm that by late gestation the production of granule cells and their precursors has relocated to the migratory pathway and the dentate hilus (Altman and Bayer, 1990; Bagri et al., 2002). Further support for this idea comes from analysis of dentate morphogenesis in primates. The extended gestational period for neurons allows studies of granule cell production in primates to show clearly that at early gestational stages granule neurons are produced in the ventricular zone and that these cells populate the dentate gyrus by radial migration but that by later gestation, all production of granule neurons continues locally in the hilus (Nowakowski and Rakic, 1979, 1981; Rakic and Nowakowski, 1981).

\section{LRP6 and Lef1 mutant mice have disorganization of the dentate radial glial network}

Dentate granule cell migration is a form of radial migration using a specialized network of radial glial fibers extending from the neuroepithelium to establish a scaffolding giving the dentate granule cell layer its distinct horseshoe shape (Eckenhoff and Rakic, 1984). Because the robustness of the migratory pathway in LRP6, Lef1, and double mutant mice was affected, we examined the integrity of the radial glial network. Although GFAP has traditionally been thought to be an astrocytic marker, in primates and in selected rodent brain regions (the dentate gyrus and cerebellum) radial glial cells also express GFAP (Levitt et al., 1981; Rickmann et al., 1987; Liu et al., 2000), and the human GFAP promoter drives expression broadly in rodent radial glial cells (Malatesta et al., 2003). In LRP6, Lef1 and double mutant mice GFAP antibody staining had lost the normal horseshoe shaped scaffolding structure with loss of the upper blade of the dentate granule cell layer in all the mutant genotypes to a similar degree (Fig. 3A-E). This was despite the normal presence of Reelin expressing Cajal-Retzius cells in the molecular layer of the dentate gyrus (Fig. $1 C, C^{\prime}$ ). Because recent studies have shown that radial glia are neuronal progenitors during embryogenesis (Noctor et al., 2001, 2002), early postnatal life (Tramontin et al., 2003), and in adulthood (Doetsch et al., 1999; Seri et al., 2001), it is difficult to separate our findings on the radial glial cells and the dentate precursor cells, and it seems most likely that our data implicate Wnt signaling as a factor in regulating the dynamics of the production and maintenance of these overlapping populations of cells in the dentate. 


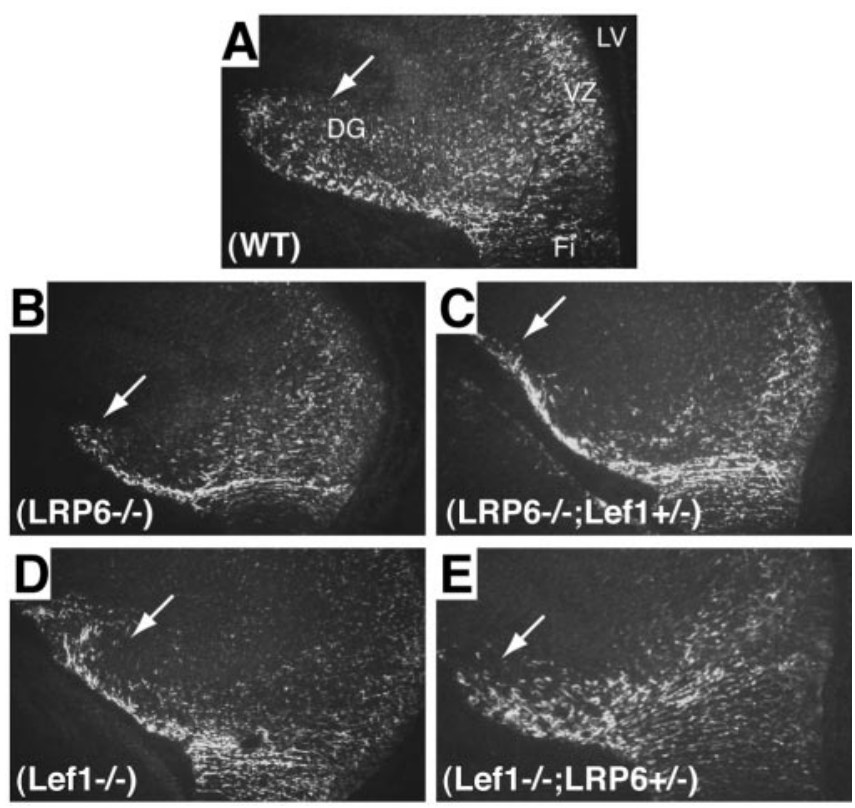

Figure 3. Radial glial scaffolding in Wnt signaling mutants. GFAP antibody staining of coronal sections at E17.5. Arrows indicate the proper position of the dentate anlage. Note that mutants lack the radial glial scaffolding in the mutants of all genotypes. LV, Lateral ventricle; DG, dentate gyrus; Fi, fimbria; VZ, ventricular zone.

\section{Early defects in the dentate neuroepithelium and dentate precursor cells}

To better understand the genesis of the failure to produce granule cells, granule precursors, and dentate radial glia, we examined early developmental consequences of loss of Wnt signaling in the dentate neuroepithelium before the appearance of differentiated dentate granule neurons and before the establishment of the displaced proliferative zones in the migratory pathway and hilus. Central to this analysis is the availability of markers that identify regions of the hippocampal neuroepithelium that contribute to various domains of the hippocampus. Using our anti-Proxl antiserum in fresh frozen tissue, we noted that at E13.5 that Prox1 staining marks the region of hippocampal neuroepithelium immediately above the cortical hem, the area that produces the future dentate granule neurons and dentate precursors (Altman and Bayer, 1990; Bagri et al., 2002). Because at later ages, stronger Proxl staining is a faithful marker of dentate granule fate, we believe that this region is the dentate neuroepithelium, from which future granule cells and their precursors arise. In LRP6 mutants this domain is smaller (Fig. 4A,B). Within this neuroepithelial domain, unlike at later stages, all acutely dividing precursors express Prox1, and these double-labeled cells are reduced in conjunction with the reduced distribution of Prox1 in the ventricular zone (Fig. 4C,D). Quantitation of this data showed a statistically significant $(p<0.01) 40 \%$ decrease in the number of these double-labeled cells, implying a corresponding decrease in the size of the dentate neuroepithelium (Fig. 4E).

\section{Discussion}

Our study shows that defects in the $\mathrm{Wnt} / \beta$-catenin signaling pathway lead to dramatic decrease in the production of one of the principal cell types of the mammalian hippocampus and that disorganization of the radial glial scaffolding accompanies this defect. These findings are consistent with a requirement for Wnt signaling in specifying or regulating the size of the initial region of neuroepithelium designated to produce granule cells and also

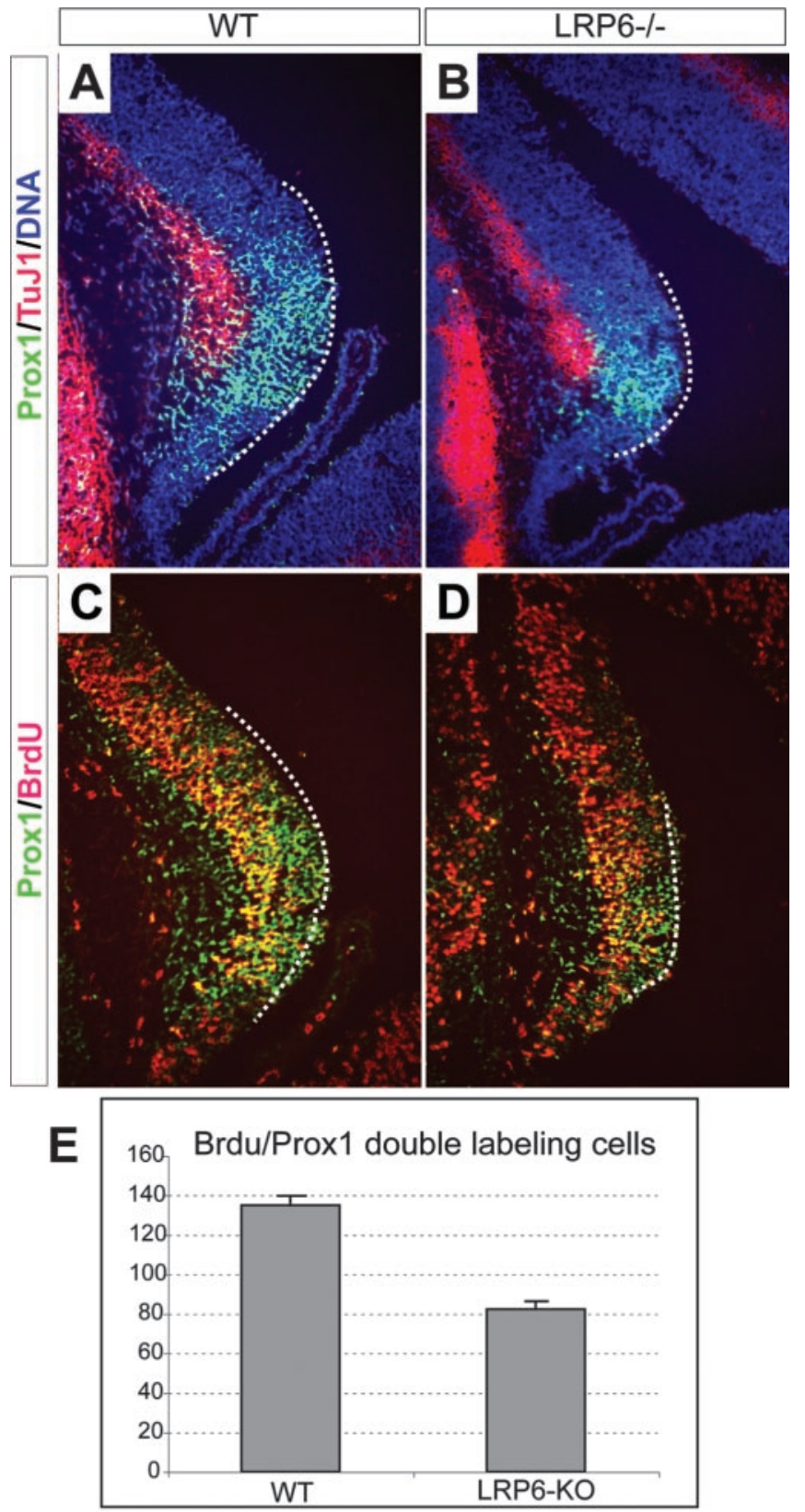

Figure 4. The ventricular zone region containing dentate precursors at mid-gestation (E13.5) is reduced in LRP6 mutants. $A$ and $B$ show Prox1/TuJ1/DNA triple-labeling. TuJ1 is a mouse monoclonal specific for a neuronal form of $\beta$-tubulin that is expressed in the cortical plate. The dotted line indicates the region of Prox 1 staining in the ventricular zone that is concentrated above the cortical hem. (and D show Prox1/BrdU double-labeling. In the dentate ventricular zone, all the BrdU-labeled cells in the ventricular zone express Prox1. $E$ shows the counting data for BrdU/Prox1 double-labeled cells.

with continued roles for Wnt signaling in dentate granule cell production caused by effects on precursor proliferation, migration, and radial glial scaffold structure. These multistage effects on granule cell production lead to an aggregate loss of the majority of differentiated granule cell neurons in the dentate.

\section{Relationship between LRP6/Lef1 and Wnt3A phenotypes}

Why do LRP6 and Lef1 mutants have defects largely limited to the dentate gyrus within the hippocampus? The most plausible explanation is a combination of timing and developmental compensation. The Wnt3A mutant phenotype is believed to be caused 
by a failure of expansion of the earliest group of hippocampal precursors between E9.5 and E10.5 (Lee et al., 2000). After this early defect, even the later expression of other Wnt family members in the cortical hem of Wnt3A mutants is unable to rescue the production of any of the types of hippocampal neurons. LRP6 and Lef1 are both probably partially compensated for by other family members, LRP5 and Tcfs, respectively. In the case of Lef1, some of the specificity for the dentate may be attributable to selective expression of Lef1 in the dentate neuroepithelium, whereas other Tcf/Lef family members are more widely expressed (Galceran et al., 2000), however, in the case of LRP6 (and LRP5), specific expression domains are not likely to be involved because both are expressed ubiquitously in the brain (C. J. Zhou and S. J. Pleasure, unpublished observations). Our finding of additive effects of LRP6 and Lef1 mutant alleles strongly indicates compensation within the canonical Wnt pathway, perhaps this compensation is more effective at early ages so that neither defect approaches the Wnt3A mutant in severity.

\section{Continuum between radial glial cells and dentate precursors}

Recent studies have shown that adult neural stem cells in the subventricular zone and dentate gyrus share some characteristics with astrocytes and radial glial cells (Doetsch et al., 1999; Seri et al., 2001) and that during cortical development most excitatory neurons descend from precursors with radial glial features (Malatesta et al., 2003). Although it is likely that there are substantial differences between the radial glial-like precursors of the embryonic brain and the astrocyte-like precursors of the adult dentate gyrus, these cells do share some features that suggest similarities. Our data show that Wnt signaling is involved in regulating the number of precursors setting up the dentate anlage and the radial glial network required for normal morphogenesis of the dentate. This is consistent with the now appreciated intimate and overlapping relationship between these cell populations. Because other mutants with defects in dentate morphogenesis have separable effects on radial glia or precursor cells (Liu et al., 2000; Forster et al., 2002), this implies that other aspects of the development of these cells are separable. Our data indicate that Wnts have roles in virtually all aspects of prenatal dentate gyrus development. Whether Wnts have similar roles in regulating the continued production of dentate granule cells postnatally will only be addressed using approaches that are able to bypass the early developmental defects in the dentate gyrus.

\section{Similarities between the hippocampal neuroepithelium and the dorsal spinal cord}

The hippocampus is derived from the medial wall of the telencephalon during embryonic life, which, because of the morphogenic events of cortical formation, is analogous to the most dorsal structures of the neural tube (Wilson and Rubenstein, 2000). The development of the dorsal neural tube is regulated by the roofplate, which shares features with the cortical hem (Grove et al., 1998; Lee and Jessell, 1999; Monuki et al., 2001). Previous studies implicated Wnt signaling from the roofplate or cortical hem primarily in regulation of precursor proliferation in both the dorsal neural tube and medial cortical wall (Ikeya et al., 1997; Lee et al., 2000; Megason and McMahon, 2002). Recently a reexamination of the spinal cord phenotype of Wnt1/Wnt3A double mutant mice and ectopic expression studies in chick spinal cord showed that Wnt signaling controls dorsal spinal interneuron development by controlling the distribution of markers that identify precursor domains required for dorsal interneuron specification adjacent to the roofplate (Muroyama et al., 2002; Helms and
Johnson, 2003). The hippocampus has a simplified developmental plan that is analogous to the spinal cord, in that distinct neuronal types are primarily generated by separate neuroepithelial domains. This idea is supported by our study showing a molecularly distinct dentate granule germinative domain and previous retroviral lineage analysis, showing separate clonal origins for pyramidal and granule neurons (Grove et al., 1992). Thus, dentate granule cells are analogous to dorsal spinal interneurons because they are generated by a precursor domain directly adjacent to the roofplate/hem domain. Our data, showing that LRP6 and Lef1 mutants have decreased dentate neuroepithelial precursor domains reveals further important similarities between the developmental plan of the hippocampus and dorsal spinal cord and reiterate the importance of Wnt signaling in dorsal neuronal specification.

\section{References}

Altman J, Bayer SA (1990) Mosaic organization of the hippocampal neuroepithelium and the multiple germinal sources of dentate granule cells. J Comp Neurol 301:325-342.

Amaral DG (1978) A Golgi study of cell types in the hilar region of the hippocampus in the rat. J Comp Neurol 182:851-914.

Bagri A, Gurney T, He X, Zou YR, Littman DR, Tessier-Lavigne M, Pleasure SJ (2002) The chemokine SDF1 regulates migration of dentate granule cells. Development 129:4249-4260.

Doetsch F, Caille I, Lim DA, Garcia-Verdugo JM, Alvarez-Buylla A (1999) Subventricular zone astrocytes are neural stem cells in the adult mammalian brain. Cell 97:703-716.

Eckenhoff MF, Rakic P (1984) Radial organization of the hippocampal dentate gyrus: a Golgi, ultrastructural, and immunocytochemical analysis in the developing rhesus monkey. J Comp Neurol 223:1-21.

Forster E, Tielsch A, Saum B, Weiss KH, Johanssen C, Graus-Porta D, Muller U, Frotscher M (2002) Reelin, Disabled 1, and beta 1 integrins are required for the formation of the radial glial scaffold in the hippocampus. Proc Natl Acad Sci USA 99:13178-13183.

Galceran J, Miyashita-Lin EM, Devaney E, Rubenstein JL, Grosschedl R (2000) Hippocampus development and generation of dentate gyrus granule cells is regulated by LEF1. Development 127:469-482.

Grove EA, Kirkwood TB, Price J (1992) Neuronal precursor cells in the rat hippocampal formation contribute to more than one cytoarchitectonic area. Neuron 8:217-229.

Grove EA, Tole S, Limon J, Yip L, Ragsdale CW (1998) The hem of the embryonic cerebral cortex is defined by the expression of multiple Wnt genes and is compromised in Gli3-deficient mice. Development 125:2315-2325.

Hari L, Brault V, Kleber M, Lee HY, Ille F, Leimeroth R, Paratore C, Suter U, Kemler R, Sommer L (2002) Lineage-specific requirements of betacatenin in neural crest development. J Cell Biol 159:867-880.

Helms AW, Johnson JE (2003) Specification of dorsal spinal cord interneurons. Curr Opin Neurobiol 13:42-49.

Ikeya M, Lee SM, Johnson JE, McMahon AP, Takada S (1997) Wnt signalling required for expansion of neural crest and CNS progenitors. Nature 389:966-970.

Lee KJ, Jessell TM (1999) The specification of dorsal cell fates in the vertebrate central nervous system. Annu Rev Neurosci 22:261-294.

Lee SM, Tole S, Grove E, McMahon AP (2000) A local Wnt-3a signal is required for development of the mammalian hippocampus. Development 127:457-467.

Levitt P, Cooper ML, Rakic P (1981) Coexistence of neuronal and glial precursor cells in the cerebral ventricular zone of the fetal monkey: an ultrastructural immunoperoxidase analysis. J Neurosci 1:27-39.

Liu M, Pleasure SJ, Collins AE, Noebels JL, Naya FJ, Tsai MJ, Lowenstein DH (2000) Loss of BETA2/NeuroD leads to malformation of the dentate gyrus and epilepsy. Proc Natl Acad Sci USA 97:865-870.

Malatesta P, Hack MA, Hartfuss E, Kettenmann H, Klinkert W, Kirchhoff F, Gotz M (2003) Neuronal or glial progeny: regional differences in radial glia fate. Neuron 37:751-764.

Maretto S, Cordenonsi M, Dupont S, Braghetta P, Broccoli V, Hassan AB Volpin D, Bressan GM, Piccolo S (2003) Mapping Wnt/beta-catenin 
signaling during mouse development and in colorectal tumors. Proc Natl Acad Sci USA 100:3299-3304.

Megason SG, McMahon AP (2002) A mitogen gradient of dorsal midline Wnts organizes growth in the CNS. Development 129:2087-2098.

Monuki ES, Porter FD, Walsh CA (2001) Patterning of the dorsal telencephalon and cerebral cortex by a roof plate-Lhx2 pathway. Neuron 32:591-604.

Muroyama Y, Fujihara M, Ikeya M, Kondoh H, Takada S (2002) Wnt signaling plays an essential role in neuronal specification of the dorsal spinal cord. Genes Dev 16:548-553.

Noctor SC, Flint AC, Weissman TA, Dammerman RS, Kriegstein AR (2001) Neurons derived from radial glial cells establish radial units in neocortex. Nature 409:714-720.

Noctor SC, Flint AC, Weissman TA, Wong WS, Clinton BK, Kriegstein AR (2002) Dividing precursor cells of the embryonic cortical ventricular zone have morphological and molecular characteristics of radial glia. J Neurosci 22:3161-3173.

Nowakowski RS, Rakic P (1979) The mode of migration of neurons to the hippocampus: a Golgi and electron microscopic analysis in foetal rhesus monkey. J Neurocytol 8:697-718.

Nowakowski RS, Rakic P (1981) The site of origin and route and rate of migration of neurons to the hippocampal region of the rhesus monkey. J Comp Neurol 196:129-154.
Pinson KI, Brennan J, Monkley S, Avery BJ, Skarnes WC (2000) An LDLreceptor-related protein mediates Wnt signalling in mice. Nature 407:535-538.

Pleasure SJ, Collins AE, Lowenstein DH (2000) Unique expression patterns of cell fate molecules delineate sequential stages of dentate gyrus development. J Neurosci 20:6095-6105.

Rakic P, Nowakowski RS (1981) The time of origin of neurons in the hippocampal region of the rhesus monkey. J Comp Neurol 196:99-128.

Rickmann M, Amaral DG, Cowan WM (1987) Organization of radial glial cells during the development of the rat dentate gyrus. J Comp Neurol 264:449-479.

Seri B, Garcia-Verdugo JM, McEwen BS, Alvarez-Buylla A (2001) Astrocytes give rise to new neurons in the adult mammalian hippocampus. J Neurosci 21:7153-7160.

Tamai K, Semenov M, Kato Y, Spokony R, Liu C, Katsuyama Y, Hess F, Saint-Jeannet JP, He X (2000) LDL-receptor-related proteins in Wnt signal transduction. Nature 407:530-535.

Tramontin AD, Garcia-Verdugo JM, Lim DA, Alvarez-Buylla A (2003) Postnatal development of radial glia and the ventricular zone (VZ): a continuum of the neural stem cell compartment. Cereb Cortex 13:580-587.

Wilson SW, Rubenstein JL (2000) Induction and dorsoventral patterning of the telencephalon. Neuron 28:641-651. 